\title{
Self-Reported Acalculia as a Presenting Symptom of Non-Dominant Right Parietal Glioblastoma Multiforme
}

\author{
Kai Miller ${ }^{1}$, Linda W. Xu ${ }^{2}$, Andrew Trister ${ }^{3}$, Jason Rockhill ${ }^{3}$, Daniel L. Silbergeld ${ }^{4}$ \\ 1. Department of Neurosurgery, Stanford University School of Medicine 2. Department of Neurosurgery, \\ Stanford University 3. Department of Radiation Oncology, University of Washington, Seattle, WA 4. \\ Department of Neurosurgery, University of Washington School of Medicine, Seattle, WA
}

$\square$ Corresponding author: Kai Miller, kjmiller@gmail.com

Disclosures can be found in Additional Information at the end of the article

\section{Abstract}

Acalculia, the acquired inability to perform simple mathematical operations, is classically associated with lesions to the left angular and supramarginal gyri. In this case report, we present a pair of right-handed patients whose presenting complaint was acalculia in the absence of finger agnosia, agraphia, or language deficit. Both patients had WHO grade IV glioblastoma multiforme tumors in the right superior parieto-occipital cortex. We hypothesize that the acalculia with abnormal tumor locale may be related to the way that these two individuals process mathematical problems. Both were extremely highly functioning - one being a university professor and the other a $\mathrm{CEO}$ of a financial institution. It is known that for many people simple mathematical operations are a function primarily of internal auditory recital and working memory, and in others, equations obey a type of sentence structure linked to the same cognitive mechanisms as writing. However, some individuals form internal spatial/structural diagrams for simple equations and solve mathematical solutions by spatial manipulation. We hypothesize that the high-functioning patients in our report shared a common mechanism for solving simple arithmetic problems, involving the spatial manipulation of mathematical objects in an internal space that is represented and manipulated in non-dominant right parietal cortex - and therefore their mathematical ability was disrupted by the invasion of tumor into this region and associated sequelae (tissue invasion, edema, mass effect).

Categories: Radiation Oncology, Neurosurgery

Keywords: acalculia, parietal cortex, non-dominant hemisphere, glioblastoma, mathematical processing

\section{Published 05/15/2012}

\section{(๑) Copyright 2012}

Miller et al. This is an open access article distributed under the terms of the Creative Commons Attribution License CC-BY 3.0., which permits unrestricted use, distribution, and reproduction in any medium, provided the original author and source are credited.

\section{Introduction}

In a now famous case from 1848, a New England railroad construction foreman, Phinneas Gage, suffered from an accident where a large metal pike pierced through his frontal lobe. He survived the incident, and suffered from personality change characterized by loss of inhibition [1]. Although the cognitive changes were vague in his case, it marked the beginning of a tradition where distinct anatomical lesions could be correlated with reproducible functional deficit. The first systematic study of this kind was published in 1861 by the French physician Paul Broca, who described how lesions to the inferior-lateral left frontal lobe produced aphasias (hence, "Broca's area") [2]. Subsequent study of lesions informed early understanding of a myriad of functional modalities in the brain, including memory, vision, motor, and sensory organization [3-4]. Lesion-related loss of mathematical ability was first described by Lewandowsky and 
Stadelmann in 1908, and eventually came to be called "acalculia" [5]. In the earliest clear delineation of acalculia, Peritz described the left (dominant) angular gyms as being the brain's "calculation center", and clearly stated that corresponding right-sided lesions did not produce a similar effect [6]. The vast majority of subsequent lesion-based acalculia cases have been associated with dominant hemisphere pathology [5, 7, 8-10]. Although some subsequent cases have described right-parietal lesions inducing acalculia [11] recent electrocortical stimulation study showed complete lateralization of numerical processing to the dominant hemisphere [12]. In this report, we describe a pair of right-handed patients who presented with acalculia. These cases are of exceptional interest for several reasons: both patients had right parietal glioblastoma; both patients were extremely highly functioning - one a university professor and the other a CEO of a financial institution; and both self-identified basic arithmetic deficits as an initial symptom.

\section{Case Presentation}

\section{Patient A}

One night, patient A (Table 1), a 68-year-old right-handed woman who had been a pianist for 60 years, was listening to Moonlight Sonata and found herself unable to play it at the piano. Concerned, she then attempted to play from children's beginner books, but was unable to remember the notes. She then sat down and tried simple math calculations, but was unable solve them. A visit to her neurologist resulted in an MRI, revealing a ring-enhancing, $-4 \times 5 \mathrm{~cm}$ right parietal lesion, above the parieto-occipital sulcus and posterior to the post central sulcus, involving primarily the superior parietal lobule and the precuneus (Figure 1). Post-resection histology of the tumor revealed it to be glioblastoma (WHO grade IV).

\section{Patient B}

As an executive in a large financial firm, Patient B, a 65-year-old right-handed male (Table 1), regularly performed simple calculations in the course of his day-to-day life. He began to notice some difficulty with these, but was not concerned initially. Soon after the symptoms started, he left for a several week vacation in Africa, where he initially noticed only that his day-to-day mathematical and cognitive abilities gradually deteriorated, to the point that change in his affect was apparent to his family upon his return. When he presented to his local hospital, an MRI revealed a large, multifocal $-7 \mathrm{x} 4 \mathrm{~cm}$ lesion in his right parietooccipital cortex lesion with extension anteriorly to the central sulcus (Figure 1). He also had a small $1 \mathrm{~cm}$ lesion in the left splenium of the corpus callosum, not involving the gyral anatomy. Post-resection histology of the tumor revealed it to be glioblastoma (WHO grade IV). 


\section{Cureus}

\begin{tabular}{|lll|l|}
\hline & Patient A & Patient B \\
\hline Age & 68 & 65 \\
Sex & F & M \\
Handedness & R & R \\
Profession & University professor & Attended prestigious law school; multimillionaire who \\
started own investment firm.
\end{tabular}

\section{TABLE 1: Patient Characteristics}

Note that in both patients, many symptoms improved post-resection/steroids/radiation, but mathematical ability did not return to baseline. Curiously, neither could articulate what internal method they used to perform simple math.

\section{Discussion}

From a clinical perspective, what makes these cases unusual beyond the lesion site is that the inability to perform basic calculations was an initial self-reported phenomenon, rather than initially being part of a classic constellation of deficits. For example, Gerstmann syndrome is a constellation of deficits associated with angular gyrus area lesions or electrocortical stimulation, of which acalculia is an element, but also includes finger agnosia, right/left disorientation, and agraphia [9-10]. Acalculia is often also found with concomitant aphasia [13]. On initial presentation, the patients noted none of these additional symptoms. Furthermore, both patients were right-handed and did not have any language deficit, so, in their case, the sites of the lesions in the right hemisphere are the non-dominant side.

Learning and performing mathematical operations involves multiple brain modalities related to working memory, language, spatial reasoning, and phonation [14-18]. Furthermore, simple mathematical calculations can be performed in a variety of ways. In some people, simple operations are performed 'automatically' and are a function primarily of internal auditory recital and working memory, while in others, equations obey a type of sentence structure linked to the same cognitive mechanisms as writing (and therefore, acalculic lesions are linked to agraphia). In yet another group, individuals form spatial/structural diagrams, and mathematical solutions are obtained by spatial manipulation [15]. Therefore, the relative impact of a lesion in a specific brain area might differentially affect individuals depending on their strategy for 


\section{Cureus}

mathematical calculation.
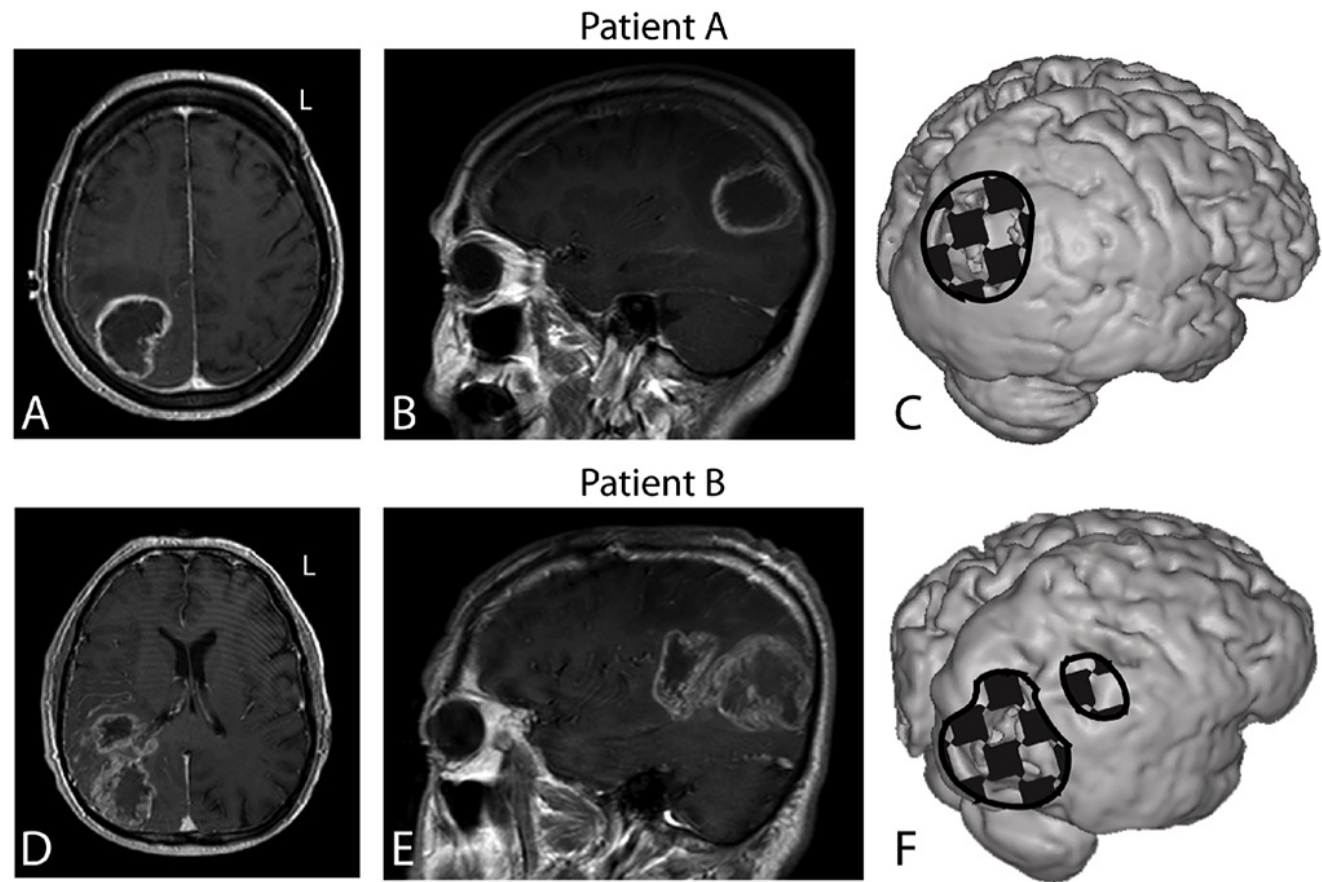

FIGURE 1: Imaging of the two patients at time of presentation.

(A-B) Axial and sagittal T1-MRI with gadolinium contrast for patient A. (C) A reconstruction of the cortical surface, with the approximate surface-intersection of the lesion shown with the patchwork mask. Note the sulcal effacement. (D-F) As in A-C, for patient B.

Rosseli and Ardila, in a comprehensive lesion study, found that there were calculative deficits associated with right parietal lesions, and that these were associated with spatial neglect and errors in spatial judgment, termed spatial acalculia [9]. Patient B, in particular, developed a spatial neglect in numeric recognition, noticed when he could not read the clock face. In contrast to our two patients, who identified the mathematical difficulty themselves, the patients of Rosselli and Ardila were oblivious to the errors that they made.

It has been demonstrated that there are different ways that individuals solve very simple mathematical problems wherein exact solutions are linked to the language process that the mathematics were learned in. Dehaene and colleagues demonstrated that when bilingual individuals solve basic mathematical problems exactly, their cognitive processes as well as response times were faster in their original mother tongue. When they solved problems approximately (i.e. "ballpark estimates"), there was no language-dependent difference in the response times or processing speeds [19]. The Dehaene group later showed, by examining Amazonian tribes without a comparable mathematical formalization that our intrinsic system for basic computation obeys logarithmic scaling (a defect in which might be inferred for patient B, who could add $2+5$, but not $5+2$ ), whereas formalized arithmetic obeys linear scaling [20]. If these complementary systems have anatomically distinct substrates in the brain, as has been demonstrated for retrieval of rote verbal arithmetic facts versus active manipulation of numerical quantities [21], then perhaps individuals like our two patients, presenting with acalculia in the setting of right parietal lesions, represent individuals who solve simple arithmetic problems atypically (rather than having atypical cortical functional organization). We hypothesize that the high-functioning patients in our study shared a common mechanism for solving simple arithmetic problems, involving the spatial manipulation of mathematical 
objects in an internal space, that this space is represented and manipulated in the nondominant right parietal regions of cortex, and therefore is disrupted by the invasion of developing tumor.

\section{Conclusions}

N/A

\section{Additional Information \\ Disclosures}

Human subjects: All authors have confirmed that this study did not involve human participants or tissue. Conflicts of interest: In compliance with the ICMJE uniform disclosure form, all authors declare the following: Payment/services info: All authors have declared that no financial support was received from any organization for the submitted work. Financial relationships: All authors have declared that they have no financial relationships at present or within the previous three years with any organizations that might have an interest in the submitted work. Other relationships: All authors have declared that there are no other relationships or activities that could appear to have influenced the submitted work.

\section{References}

1. Harlow JM: Recovery from the passage of an 12. iron bar through the head . Publ Mass Med Soc. $1868,2: 327$.

2. Broca P: Remarques sur le siege de la faculte du langage articule, suivies dune observation 13. d'aphemie (perte de la parole). Bulletin de la Societe Anatomique. 1861, 6:330-357.

3. Ribot T: Diseases of memory. Appleton, 1887.

4. Clark D: Brain Lesions and Functional Results. American Journal of Insanity. 1881, 37:241.

5. Boller F, Grafman J: Acalculia: Historical development and current significance . Brain Cogn. 1983, 2:205-223.

6. Peritz G: Zur Pathopsychologie des rechnens. Journal of Neurology. 1918, 61:234-340.

7. Takayama Y, Sugishita M, Akiguchi I, et al.: Isolated acalculia due to left parietal lesion. Arch Neurol. 1994, 51:286-91.

8. McCloskey M: Cognitive mechanisms in numerical processing: evidence from acquired dyscalculia. Cognition. 1992, 44:107- 57.

9. Rosselli M, Ardila A: Calculation deficits in patients with right and left hemisphere damage . Neuropsychologia. 1989, 27:607-617.

10. Gerstmann J: Syndrome of finger agnosia, disorientation for right and left, agraphia and acalculia: Local diagnostic value. Archives of Neurology \& Psychiatry. 1940, 44:398.

11. Roux FE, Boetto S, Sacko O, et al.: Writing, calculating, and finger recognition in the region of the angular gyrus: a cortical stimulation study of Gerstmann syndrome. J Neurosurg. 2003, 99:716-27.

12. Pu S, Li YN, Wu CX, et al.: Cortical Areas Involved in Numerical Processing: An Intraoperative Electrostimulation Study. Stereotact Funct Neurosurg. 2011, 89:42-47.

13. Basso A, Burgio F, Caporali A: Acalculia, aphasia and spatial disorders in left and right braindamaged patients. Cortex. 2000, 36:265-280.

14. Baddeley A: Working memory. Science. 1992, 255:556.

15. Leonhard K: Ideokinetic aphasia and related disorders. Neurolinguistics. Lebrun Y, Hoops R (ed): Swets and Zeitlinger, Lisse, Netherlands; 1979. 9:11-77.

16. Swanson HL, Sachse-Lee C: Mathematical problem solving and working memory in children with learning disabilities: both executive and phonological processes are important. J Exp Child Psychol. 2001, 79:294-321.

17. Geary DC, Hoard MK, Byrd-Craven J, et al.: Strategy choices in simple and complex addition: Contributions of working memory and counting knowledge for children with mathematical disability. J Exp Child Psychol. 2004, 88:121-51.

18. Graziano AB, Peterson M, Shaw GL: Enhanced learning of proportional math through music 


\section{Cureus}

training and spatial-temporal training. Neurol Res. 1999, 21:139-52.

19. Dehaene, S: Sources of Mathematical Thinking: Behavioral and Brain-Imaging Evidence . Science. 1999, 284:970-974.

20. Dehaene S, Izard V, Spelke E, et al.: Log or Linear? Distinct Intuitions of the Number Scale in Western and Amazonian Indigene Cultures. Science. 2008, 320:1217-1220.

21. Dehaene S, Cohen L: Cerebral pathways for calculation: Double dissociation between rote verbal and quantitative knowledge of arithmetic. Cortex. 1997, 33:219-250. 